\title{
BOA valence bonding with f-character in highly coordinated actinides
}

\author{
F. L. Carter \\ Naval Research Laboratory, Washington, D.C. 20375, U.S.A.
}

\begin{abstract}
Résumé. - L'incorporation de caractère $f$ aux orbitales bidirectionnelles augmente leur flexibilité de manière significative. Les orbitales $\mathrm{Cf}$ et $\mathrm{G} f$ qui en résultent sont utilisées pour l'étude des cas courants de coordination élevée dans les actinides. La méthode des liaisons de valence implique la séparation des orbitales radiales $\mathrm{d}$ et $\mathrm{f}$ en liaisons hybrides à la fois contractées (localisées) et dilatées (délocalisées).
\end{abstract}

\begin{abstract}
The addition of $f$ character to bidirectional orbitals enhances their flexibility significantly. The resultant $\mathrm{Cf}$ and $\mathrm{G}$ orbitals are applied to some common high coordinations of the actinides. The valence bond approach implies $d$ and $f$ orbital radial splittings into bonding hybrids and either contracted localized or extended supra-valent $d$ and $f$ orbitals.
\end{abstract}

In the Bidirectional Orbital Approximation (BOA) valence bonding hybridized orbitals are characterized by two lobes of high electron density concentrated toward two different nearby atoms [1]. When an actinide has high coordination and most of the bonds formed are of bond order $\leqq 1 / 2$, then the use of bidirectional orbitals are indicated. The advantages are several : (1) bond hybridization can be easily estimated by maximizing Pauling's bond strength $S$ in the bond direction as a function of hybrid character [2] ; (2) formal charges in the formation of valence bond structures are much lower than when using normal valence bond structures ; (3) the atomic orbitals are organized into hybrids available for bonding and local orbitals suitable for unshared electrons; and (4) the approach is readily extendable [1] beyond the naive level and should account for electron correlation effects in manner superior to molecular orbital treatments. The bidirectional orbitals and termed $\mathrm{C}$ and $\mathrm{G}$ orbitals whose simplest representatives are the $p_{z}$ and $d_{z^{2}}$ orbitals, respectively.

The generalization of these orbitals involving $s, p$, $d$, and $f$ orbitals can be formulated as :

$$
\begin{aligned}
& \mathrm{Cf}=D \cdot \mathrm{p}_{z}+F \cdot \mathrm{d}_{x z}+J \cdot \mathrm{f}_{z\left(5 z^{2}-3 r^{2}\right)}+M \cdot \mathrm{f}_{z\left(x^{2}-y^{2}\right)} \\
& \mathrm{Gf}=A \cdot \mathrm{s}+B \cdot \mathrm{p}_{x}+I \cdot \mathrm{d}_{z^{2}}+K \cdot \mathrm{f}_{x\left(5 z^{2}-r^{2}\right)} .
\end{aligned}
$$

The $\mathrm{Cf}$ orbital is a generalization of the $\mathrm{Cf} 1$ and $\mathrm{Cf} 2$ orbitals employed in the discussion of the $\mathrm{Pu}_{2} \mathrm{C}_{3}$ and the $\mathrm{CrUS}_{3}$ structures, respectively $[3,4]$. The BOA method involves using the $\mathrm{Cf}$ and $\mathrm{Gf}$ orbitals in such a way that (1) all neighbours are simultaneously bonded and (2) equivalent neighbours are bonded in an equivalent manner. These give rise to orthogonality relationships between the coefficients $A, B, \ldots$ $Q$, as indicated previously for a variety of coordination spheres [5-7] without $f$ character.
Figure 1 illustrates the $\mathrm{Cf}$ and Gf orbitals for cubic close packed (CCP) coordination and the Friauf polyhedron of the Laves phases, respectively, while figure 2 shows the high strength of the unrestricted $\mathrm{Cf}$ orbital as a function of theta. (Bonding angle between the lobes $=180^{\circ}-2$ * theta.) For the CCP and icosahedral coordinations, orthogonality conditions on the six $\mathrm{Cf}$ orbitals require that $d$ character must be $50 \%$. Figure 3 illustrates the strength $S$ of these lobes for various theta values. Table $I$ indicates the various combinations of the $\mathrm{Cf}$ and $\mathrm{Gf}$ orbitals in some common actinide high coordinations.

The use of $f$ character in the Cf and Gf orbitals not only enhances the strength $S$ of the bidirectional orbitals from $\sim 2.4$ for $\mathrm{C}$ orbitals to $\sim 3$ for the $\mathrm{Cf}$ versions (see Figs. 2 and 3 ), but also provides better alignment of the main lobes with the bond axes. However, it is doubtful that the overlap integrals are proportional to the strength $S$ of such orbitals
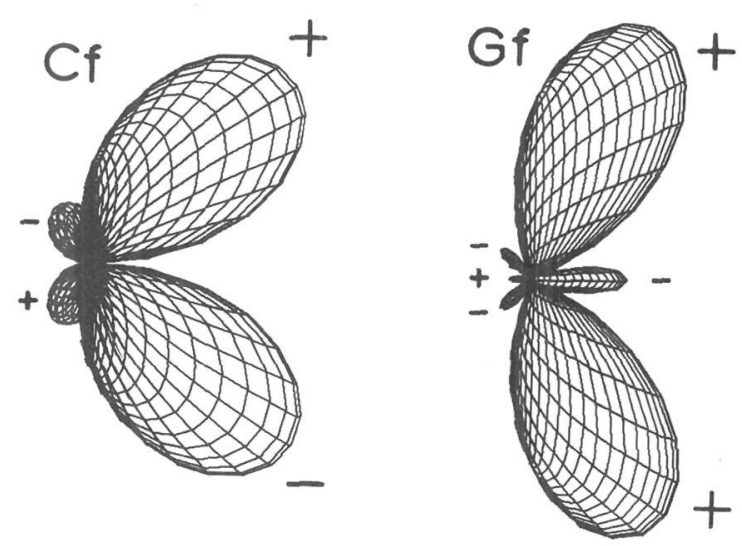

Fig. 1. - The Cf orbital with its bonding lobes $90^{\circ}$ apart is near ideal for the cubic close-packed structure, while the Gf orbital is appropriate for the $A-B$ bonds in the actinide Laves phases $A_{2}$. 
CF DRBITAL. MAX. STRENGTH

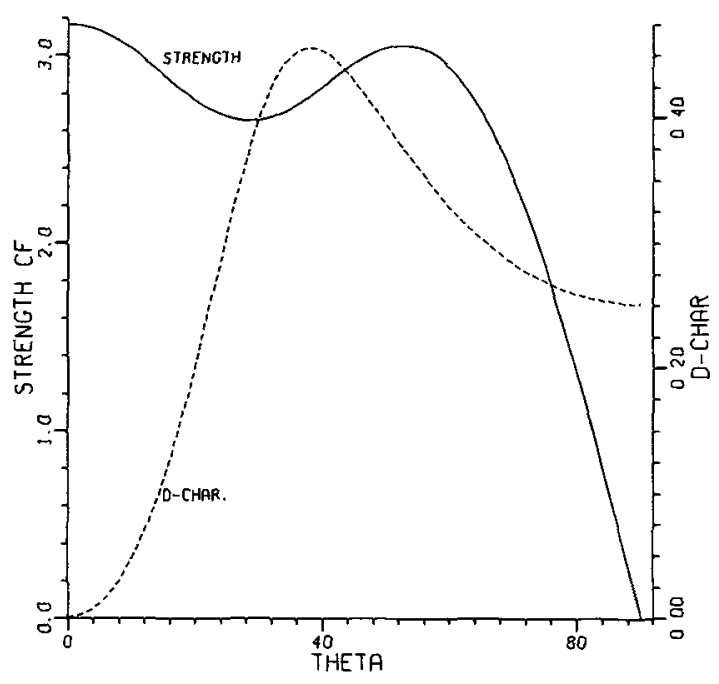

Fig. 2. - The high strength $S$ of the $\mathrm{Cf}$ orbital without restriction is indicated here. Here $\mathrm{f}$ character can be as high as $60 \%$.
CF ORBITAL. 50\% D-CHAR.

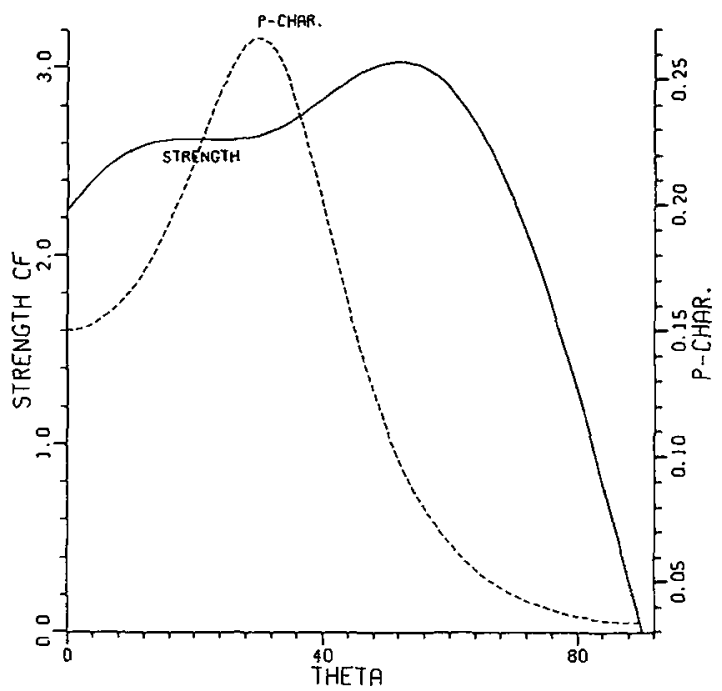

Fig. 3. - The Cf orbital under the restriction of icosahedral or CCP symmetry still has excellent strength, although the maximum $f$ character is now $34 \%$. The bonding angle between the two main lobes $=180^{\circ}-2 *$ theta.

Table I. - Bidirectional orbitals in high coordinations.

Coordination

\begin{tabular}{cr}
$\overline{6}$ & Symme \\
\cline { 2 - 2 } & 3 \\
8 & 3 \\
9 & 3 \\
12 & 32 \\
& 32 \\
14 & $\mathrm{~mm}+$
\end{tabular}

16

32

18

$\begin{array}{ccc} & \text { Gf } & \\ & \frac{}{3} & \\ & & \\ & & \\ & 3 & \text { or } \\ & 3 & \text { or } \\ & & \\ & & \\ & 4 & + \\ \text { or } & 3 & + \\ & 6 & +\end{array}$

6
Other configuration

Twisted trig. prism, octa.

+ Gf Side capped prism

$+3 \sigma \quad$ Capped prism

Icosahedron, CCP

HCP

$\mathrm{BCC}$

Friauf polyhedron, Laves phase

$+3 G$

$\mathrm{NaCl}$

${ }^{*}$ ) Note resonance restores full symmetry to site.

because of the narrowness of the $f$ lobes. Hence $f$ character is probably overestimated by this simple use of BOA.

The valence bond method assumes that in the bonding hybrids, the radial parts of the various orbitals are comparable. Due to the relativistic contractions of the $7 \mathrm{~s}$ and $7 \mathrm{p}$ orbitals and the resultant shielding effects on the $6 \mathrm{~d}$ and $5 f$ orbitals, plus the formation of bonds, the radial parts of the bonding orbitals are probably comparable in many actinide compounds. However, the orbitals not involved in bonding are likely to be more contracted, as observed for $f$ orbitals in $U_{3} \mathrm{As}_{4}$ [8] or more extended, as suggested for the $d_{z^{2}}$ orbital in $\mathrm{CrUS}_{3}[4]$.

\section{References}

[1] CARTer, F. L., Electronic Densities of State, ed. L. H. Bennett, Nat. Bur. Standards, Spec. Pub. 323 (1971) 385.

[2] Pauling, L., Nature of the Chemical Bond, 3rd ed. (Cornell Univ. Press, Ithaca, New York) 1960.

[3] Carter, F. L., Proc. 11th Rare Earth Res. Conf., eds. J. M. Haschke and H. A. Eick, Traverse City, Mich. (Oct. 1974) 36.

[4] Carter, F. L., Wolfers, P., Fillion, G., J. C. S. Dalton, in press.

[5] Carter, F. L., Proc. 5th Rare Earth Res. Conf., Ames, Iowa (1965).

[6] Carter, F. L., Proc. 7th Rare Earth Res. Conf., Coronado, Calif. (Oct. 1968) 283.

[7] Carter, F. L., Proc. 9th Rare Earth Res. Conf., ed. D. E. Field, Blacksburgh, Va., Vol. 2 (Oct. 1971) 617.

[8] Rozenfeld, B., BEDowsKa, E. and HeNkIE, Z., J. Solid State Chem. 17 (1975) 101. 